\title{
Energy spectra of secondaries in proton-proton interactions
}

\author{
S. Koldobskiy $\odot,{ }^{1,2}$ M. Kachelrie $\beta,{ }^{3}$ A. Lskavyan, ${ }^{1}$ A. Neronov, ${ }^{4,5}$ S. Ostapchenko, ${ }^{6,7}$ and D. V. Semikoz ${ }^{1,4,8}$ \\ ${ }^{1}$ National Research Nuclear University MEPHI, 115409 Moscow, Russia \\ ${ }^{2}$ Space Physics and Astronomy Research Unit and Sodankylä Geophysical Observatory, \\ University of Oulu, 90014 Oulu, Finland \\ ${ }^{3}$ Institutt for fysikk, NTNU, Trondheim N-7491, Norway \\ ${ }^{4} A P C$, Université Paris Diderot, CNRS/IN2P3, CEA/IRFU, Observatoire de Paris, \\ Sorbonne Paris Cité, 11975205 Paris, France \\ ${ }^{5}$ Astronomy Department, University of Geneva, Chemin d'Ecogia 16, 1290 Versoix, Switzerland \\ ${ }^{6}$ II. Institute for Theoretical Physics, Hamburg University, Hamburg 22761, Germany \\ ${ }^{7}$ D.V. Skobeltsyn Institute of Nuclear Physics, Moscow State University, Moscow 119992, Russia \\ ${ }^{8}$ INR RAS, 60th October Anniversary prospect 7a, Moscow 117312, Russia
}

(Received 4 October 2021; accepted 24 November 2021; published 17 December 2021)

\begin{abstract}
We compare the predictions of AAfrag for the spectra of secondary photons, neutrinos, electrons, and positrons produced in proton-proton collisions to those of the parametrizations of Kamae et al., Kelner et al. and Kafexhiu et al. We find that the differences in the normalization of the photon energy spectra reach $20 \%-50 \%$ at intermediate values of the transferred energy fraction $x$, growing up to a factor of two for $x \rightarrow 1$, while the differences in the neutrino spectra are even larger. We argue that LHCf results on the forward production of photons and neutral pions favor the use of the QGSJET-II-04m model on which AAfrag is based. The differences in the normalization have important implications in the context of multimessenger astronomy, in particular, for the prediction of neutrino fluxes, based on gamma-ray flux measurements, or regarding the inference of the cosmic ray spectrum, based on gamma-ray data. We note also that the positron-electron ratio from hadronic interactions increases with energy toward the cutoff, an effect which is missed using the average electron-positron spectrum from Kelner et al. Finally, we describe the publicly available PYTHON package aafragpy, which provides the secondary spectra of photons, neutrinos, electrons, and positrons. This package complements the AAfrag results for protons with energies above $4 \mathrm{GeV}$ with previous analytical parameterizations of particle spectra for lower energy protons.
\end{abstract}

DOI: 10.1103/PhysRevD.104.123027

\section{INTRODUCTION}

A large variety of applications in astroparticle physics relies on the precise knowledge of the production cross sections of secondary particles in hadronic interactions. A prominent example is the branch of multimessenger astronomy which aims to connect photon, neutrino and cosmic ray data $[1,2]$. While in the past simple estimates or empirical parametrizations for these cross sections were sufficient, the improved accuracy and large statistics of current and future experiments like AMS-02 [3], LHASSO [4], CTA [5] and IceCube-Gen2 [6] requires a corresponding advancement of the theoretical predictions.

There exist two main approaches to the description of hadronic production cross sections. In the first one, one parametrizes hadronic interaction data using empirical scaling laws. In spite of its convenience, the use of such parametrizations becomes dangerous when the latter are extrapolated outside the kinematical range of the data, they are based on. In particular, high-energy extrapolations into the multi-PeV range of such empirical parametrizations are generally unreliable, but are required for the correct interpretation of gamma-ray and neutrino data in the $100 \mathrm{TeV}-1 \mathrm{PeV}$ energy range. As an alternative, one can use QCD inspired Monte Carlo event generators for the description of hadronic interactions. In order to provide a fast and user-friendly tool for the computation of the production cross sections, one can first bin their results and then either interpolate or fit them. Given sufficient statistics, the former option reproduces exactly the results of the used Monte Carlo event generator, while the accuracy of the latter approach depends on the choice of appropriate fit functions. Similar to the case of empirical parametrizations, the use of such fit functions becomes dangerous when they are extrapolated outside the fit range.

While most of the QCD inspired Monte Carlo event generators used in cosmic ray physics were overall in a satisfactory agreement [7] with various data from Run I of the Large Hadron Collider (LHC), several of them have been updated by re-tuning their model parameters with LHC data. Moreover, the QGSJET-II-04m model [8,9] was further tuned in Ref. [10] to improve antiproton production 
at low energies. The results of this QGSJET-II-04m tune were used to provide AAfrag [11,12] with convenient tabulations of the production cross sections of secondary particles in proton-proton, proton-nucleus, nucleus-proton, and nucleus-nucleus reactions.

The aim of this work is to compare the predictions of various available parametrizations for the spectra of secondary photons, neutrinos, electrons, and positrons produced in proton-proton collisions to those of AAfrag. The predictions of these parametrizations are expected to vary mainly because of differences in the physics of the event generators used to produce the fit data. Moreover, parametrizations based on pre-LHC and post-LHC event generators are expected to differ significantly, especially for forward particle production and in the multi-PeV energy range. In addition, the treatment of the experimental data and the choice of the fit functions affects the predicted spectra. As a result of these differences, we find significant variations between the results of these parametrizations and we characterize the observed discrepancies. In addition, we describe and make publicly available the PYTHON package aafragpy, which provides the secondary spectra of $\gamma$-rays, neutrinos, electrons and positrons for primary proton energies in the energy range $1.5-10^{11} \mathrm{GeV}$. Since AAfrag is restricted to proton energies above $4 \mathrm{GeV}$, we complement it in this PYTHON package at lower energies with the parametrizations of Kamae et al. [13].

This article is organized as follows: We start by describing in Sec. II the main features of the parametrizations we examine. In Sec. III, we compare secondary particle production for fixed energies of the incident protons, while we discuss the case of an $1 / E^{2}$ primary proton spectrum in Sec. IV. After a presentation of the new PYTHON package aafragpy in Sec. V, we conclude.

\section{PARAMETRIZATIONS}

In addition to AAfrag, we discuss the three most commonly ${ }^{1}$ used parametrizations of Kamae et al. [13], Kelner et al. [15], and Kafexhiu et al. [14]. Their main characteristics are summarized in Table I. A drawback of QCD inspired event generators is that they cannot be used below a minimal energy, which is typically in the range of few to $100 \mathrm{GeV}$ for the energy $E_{p}$ of the projectile in the lab frame. Note that the recommended minimal energy is with 56 and $100 \mathrm{GeV}$ for PYTHIA and SIBYLL, respectively, rather high. In contrast, it was shown in Ref. [11] that QGSJET-II$04 \mathrm{~m}$ can be used down to primary energies as low as $4 \mathrm{GeV}$. All three parametrizations complement fit functions from event generators at high energies with phenomenological models at low energies. Kamae et al. used several parametrized models, including resonance-excitation components for primary energies $E_{p}$ below $52.6 \mathrm{GeV}$. Kelner et al.

\footnotetext{
${ }^{1}$ For a discussion of other models see Ref. [14].
}

TABLE I. Characteristics of the models.

\begin{tabular}{lcc}
\hline \hline & $\begin{array}{c}\text { High-energy } \\
\text { interaction model }\end{array}$ & $\begin{array}{c}\text { Range in Kinetic } \\
\text { energy, GeV }\end{array}$ \\
\hline This work & QGSJET-II-04m & $3.1-10^{11}$ \\
Kamae et al. & PYTHIA6.2 & $0.488-5.12 \times 10^{5}$ \\
Kelner et al. & SIBYLL2.1 & $100-10^{8}$ \\
Kafexhiu et al. & GEANT4.10.0 & $0.280-10^{5}$ \\
& PYTHIA8.18 & $50-10^{6}$ \\
& QGSJET-I & $100-10^{6}$ \\
& SIBYLL2.1 & $100-10^{6}$ \\
\hline \hline
\end{tabular}

proposed to use a $\delta$ function approximation for the calculation of the production spectra of secondaries at $E_{p} \leq 100 \mathrm{GeV}$ or for $E_{\mathrm{s}} / E_{p} \leq 10^{-3}$, where $E_{\mathrm{s}}$ denotes the energy of the secondary particle of interest. This approximation is restricted to the case of a power-law spectra in momentum of the proton primaries and the (invalid) assumption of a constant inelastic cross section. Kafexhui et al. relied on a compilation of experimental data below $E_{p}<2 \mathrm{GeV}$ and applied GEANT4.10.0 at intermediate energies. These latter authors considered only the production of photons. Moreover, they re-used the results for the meson spectra from Kelner et al. in the case of QGSJET-I and SIBYLL2.1, but applied different fit functions for the resulting photon spectra. Thus one can view the differences between the results of Kafexhui et al. and Kelner et al. as a measure for the deviations introduced by the fit procedure. Note also that SIBYLL2.1 was released in the year 2000, while the version of QGSJET-I used in Refs. [14,15] was published in 1997. Similarly, PYTHIA6.2 is a pre-LHC event generator dating from the year 2001.

\section{ENERGY SPECTRA FOR MONOENERGETIC PROTONS}

\section{A. Photons and neutrinos}

We start our comparison with the spectra $E^{2} \mathrm{~d} \sigma / \mathrm{d} E$ of photons and neutrinos, produced in proton-proton interactions with fixed primary energies. These spectra, which correspond to the spectral energy distribution (SED) of hypothetical sources of monoenergetic protons, are shown in Figs. 1, 2, and 3. In Fig. 1, which corresponds to the incident proton energy $E_{p}=10 \mathrm{GeV}$, we observe a $20 \%$ scatter in the model results for the photon spectra at the peak of the SED, in the photon energy range $10^{-2} E_{p}<E_{\gamma}<0.1 E_{p}$. The differences between the model predictions grow up to $\sim 50 \%$ in the high-energy tails of the SED, which reflects the lack of experimental data for very forward photon production at these energies. The calculated neutrino production spectra shown in the right panel of Fig. 1 deviate somewhat stronger, with $\sim 30 \%$ differences. In particular, the SED of AAfrag is more sharply peaked in the forward direction than the one of Kamae 


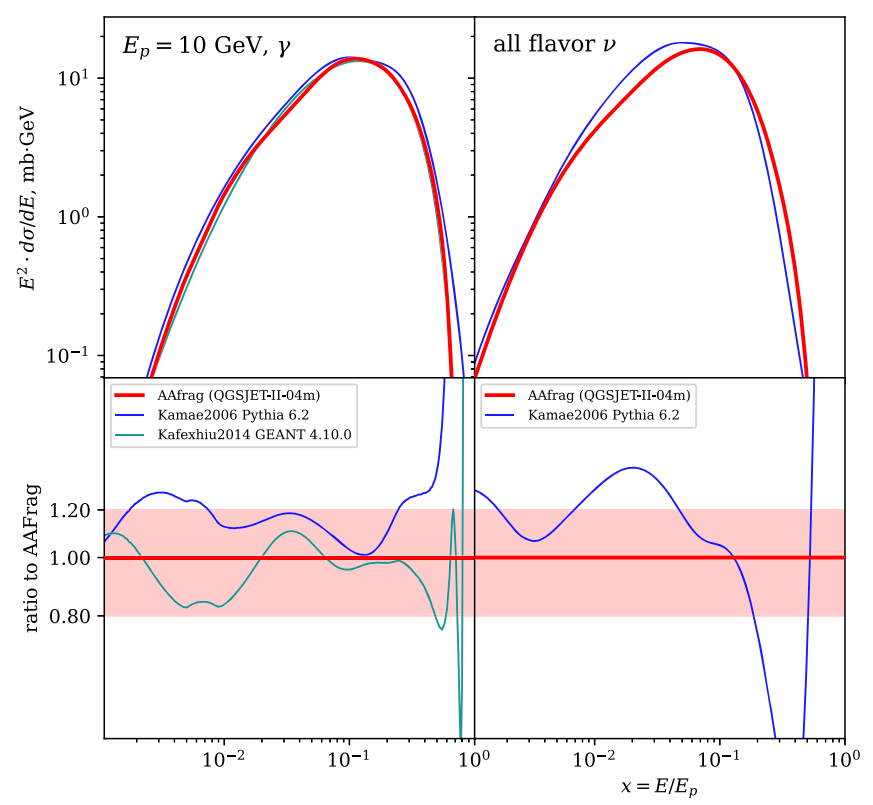

FIG. 1. Gamma-ray (left) and neutrino (right) spectra $E^{2} \mathrm{~d} \sigma / \mathrm{d} E$ as a function of the transferred energy fraction $x=E / E_{p}$, for $10 \mathrm{GeV}$ incident protons. The upper left panel compares the photon spectra calculated with AAfrag with those of Kamae et al. based on PYTHIA6.2 and Kafexhiu et al. based on GEANT4.10.0. The spectra of all-flavor neutrinos are compared to Kelner et al. and Kamae et al. The lower panels show the ratio of the spectra to the AAfrag results.

et al., which reflects the harder spectra of charged pions in QGSJET-II-04m compared to that parametrization.

Moving to higher energies, the differences between AAfrag and earlier models stay within $20 \%$ at the peak of the SED of secondary particles, cf. with Figs. 2 and 3. On the other hand, the results of the different calculations deviate much stronger in the forward direction, for $x=E_{s} / E_{p} \gtrsim 0.1$, where differences exceed $50 \%$ for some parametrizations. For the particular case of the model of Kamae et al., this deviation is related to deficiencies in their modeling of inelastic diffraction, as discussed in some detail in Ref. [16]; the corresponding contribution is visible by eye as the sharp rise of the blue lines for $E_{\gamma} \rightarrow E_{p}$ in the lower left panels of Figs. 2 and 3. On the other hand, the softer photon spectra for $E_{\gamma} \rightarrow E_{p}$ obtained in the parametrization of Kafexhui et al., based on the outdated QGSJET-I model [17], are caused by the too soft pion production spectra in that model. Overall, the increase with energy of the differences between the various predictions for the forward production of secondary particles is due to the fact that relevant experimental data have till recently been available at fixed target energies only, for $E_{p} \leq 400 \mathrm{GeV}$. This lack of experimental data has been especially unsatisfactory, because the role of forward production is greatly enhanced in the case of a steep spectrum of primary cosmic rays, as discussed in some detail in Refs. $[16,18]$; this issue will be in this work further

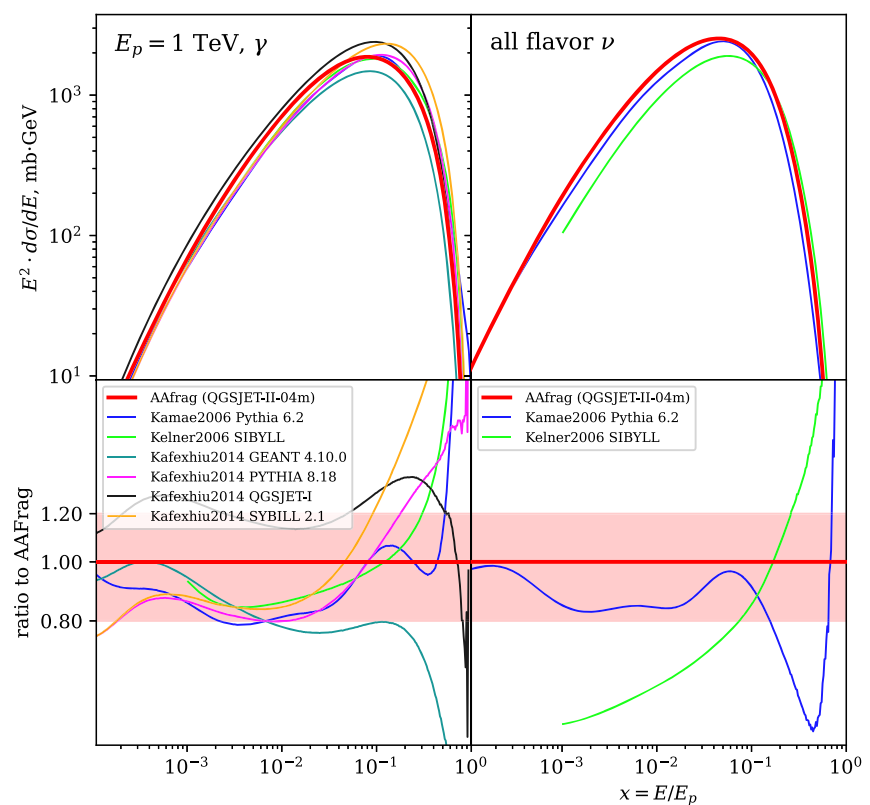

FIG. 2. Gamma-ray and neutrino spectra $E^{2} \mathrm{~d} \sigma / \mathrm{d} E$ (top) and ratios (bottom) as a function of the transferred energy fraction $x=E / E_{p}$, for $1 \mathrm{TeV}$ incident protons. The parametrizations of Kelner et al. based on SIBYLL2.1 and Kafexhiu et al. based on SIBYLL, PYTHIA and QGSJET-I are added to the analysis.

addressed in Sec. IV below. Therefore, the measurements of forward photon and neutral pion production at LHC energies, $\sqrt{s}=0.9,2.76,7$, and $13 \mathrm{TeV}$, by the LHCf experiment [19-22] are of great importance. In Fig. 4, we compare LHCf measurements (black squares with error bars) of the differential cross section of $\pi^{0}$ production at $7 \mathrm{TeV}$ c.m. energy as function of Feynman $x_{F}$ to the

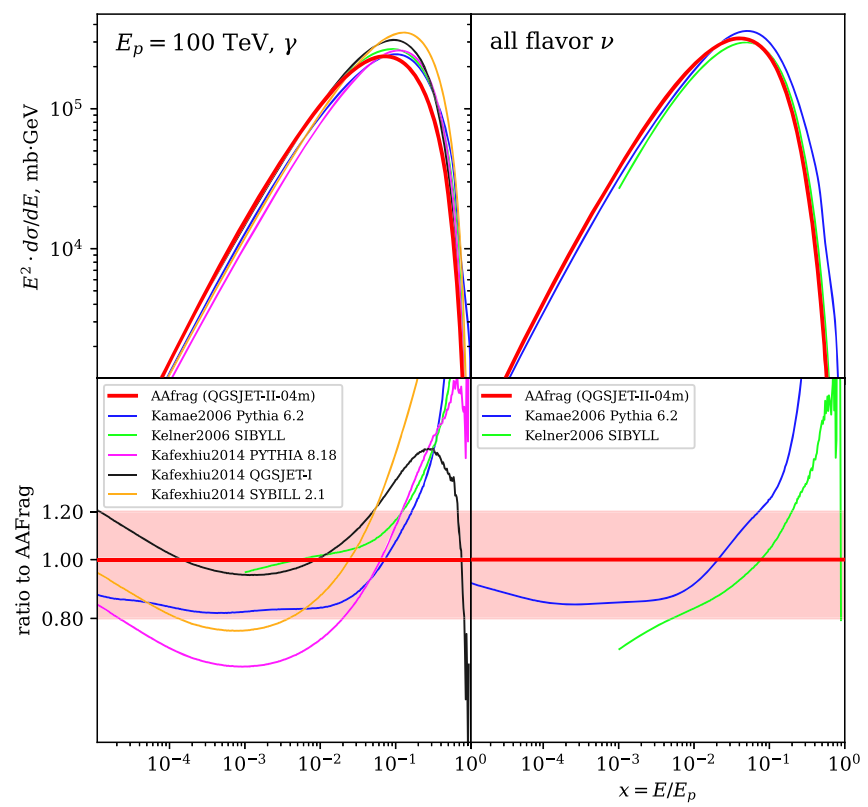

FIG. 3. Same as Fig. 2 for $100 \mathrm{TeV}$ primary protons. 


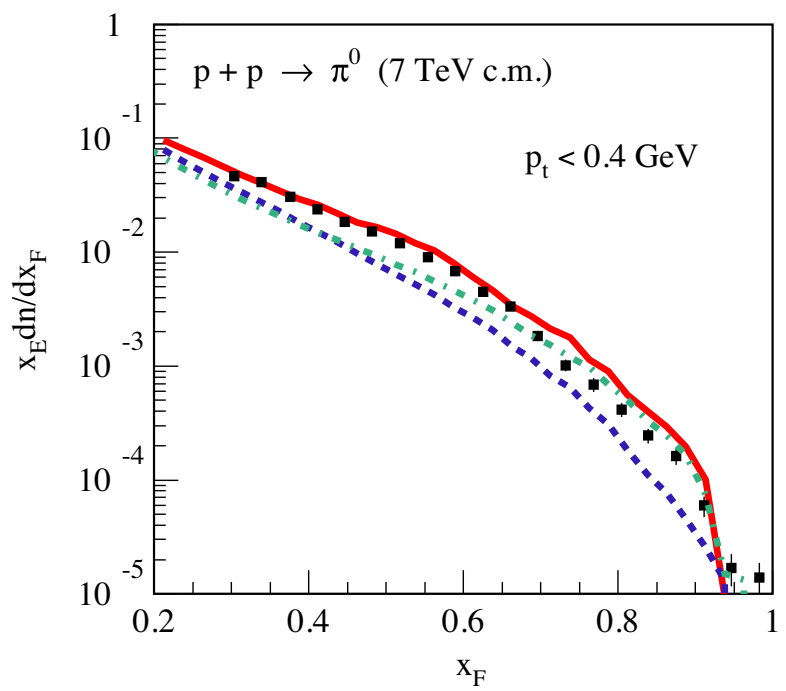

FIG. 4. Measurements of the differential cross section of $\pi^{0}$ production at $7 \mathrm{TeV}$ c.m. energy as function of Feynman $x_{F}$ by LHCf (black squares with error bars) compared to the predictions of QGSJET-II-04m (solid red), SIBYLL2.1 (dashed-dotted green) and QGSJET-I (dashed blue).

predictions of QGSJET-II-04m (solid red line), SIBYLL2.1 (dashed-dotted green) and QGSJET-I (dashed blue). It can be seen that QGSJET-II-04m describes overall the data best. The only parametrization which provides enough kinematical information that it can be compared to such measurements is the one of Kamae et al. While $7 \mathrm{TeV}$ c.m. energy is beyond the range of applicability of this parametrization, the predicted yield of photons at $900 \mathrm{GeV}$ c.m. energy is already a factor few above the LHCf measurements. Therefore, the parametrizations of Kamae et al. and Kafexhui et al. (blue, green, and magenta lines in Figs. 2 and 3) are disfavored by the LHCf data. Note also that the difference between the results of Kafexhui et al. and Kelner et al., both based on SIBYLL2.1, which is caused purely by the use of different fit functions, is as large as $20 \%$ at $x \simeq 0.1$. Moreover, we observe a rather large discrepancy with the Kelner et al. parametrization for the neutrino spectra, where both the normalization and the shape of the neutrino spectrum differ significantly from the other predictions. As a result, we expect correspondingly large errors in the predictions of the expected number of observable neutrino events from specific sources, which are based on the Kelner et al. parametrization.

Figures 1,2, and 3 also show that the predicted spectral shapes of the photon SED in the limit of small $E_{\gamma}$ differ for the various parametrizations. To clarify this point, we plot in Fig. 5 the photon production spectrum $\mathrm{d} \sigma / \mathrm{d} E$ as function of $\log (E)$ for $E_{p}=5 \mathrm{GeV}$, calculated using both AAfrag and the parametrizations. Plotted in such a way, the photon spectrum should be symmetric with respect to the energy $E_{\gamma}=m_{\pi^{0}} / 2 \simeq 67.5 \mathrm{MeV}$ [23]. While this is the case for the Kafexhiu et al. results based on GEANT4.10.0 and for AAfrag,

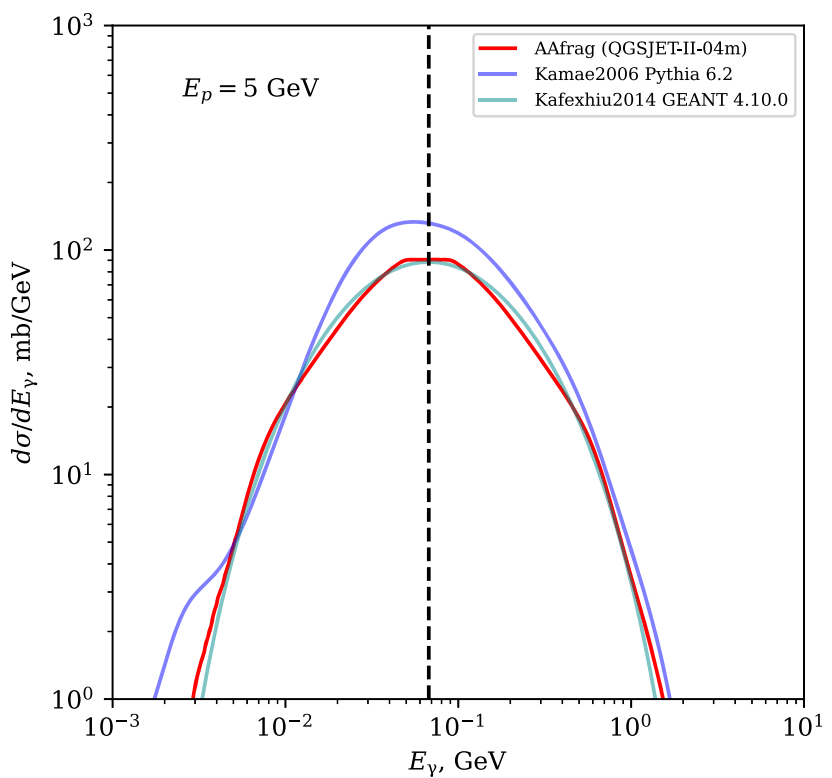

FIG. 5. Differential cross section of $\gamma$-ray production for $5 \mathrm{GeV}$ protons calculated with AAfrag, Kamae et al. and Kafexhiu et al. codes.

this symmetry is broken by the employed fit functions in the case of the parametrizations. It is worth recalling, however, that the high energy gamma-ray fluxes from astrophysical sources are dominated by the forward photon production in cosmic ray interactions (see, e.g., Ref. [18]). Therefore, the low energy part of the photon production spectra is only relevant to the calculation of photon spectra in the sub-GeV energy range, where the important contributions are coming from proton interactions at relatively low energies.

\section{B. Electrons and positrons}

In addition to photons and neutrinos, we plot in Fig. 6 the production spectra of positrons and electrons for $E_{p}=$ $100 \mathrm{GeV}$ and $1 \mathrm{TeV}$, calculated using both AAfrag and the parametrizations. Here, apart from the general differences between the various parametrizations, it is important to note that, unlike AAfrag and Kamae et al., the parametrization of Kelner et al. provides average spectra between $e^{+}$and $e^{-}$. That way, one neglects the important difference between them, which stems from significantly harder production spectra of $\pi^{+}$mesons, compared to $\pi^{-}$, in proton-proton interactions. As will be further discussed in Sec. IV B, this has important consequences for the analysis and the interpretation of experimental data on cosmic-ray fluxes of electrons and positrons.

\section{ENERGY SPECTRA FROM BROAD ENERGY DISTRIBUTIONS OF PROTONS}

The intensity $I_{a}$ of secondary particles of type $a$ is related to the intensity of primary cosmic rays $I_{p}$ as 


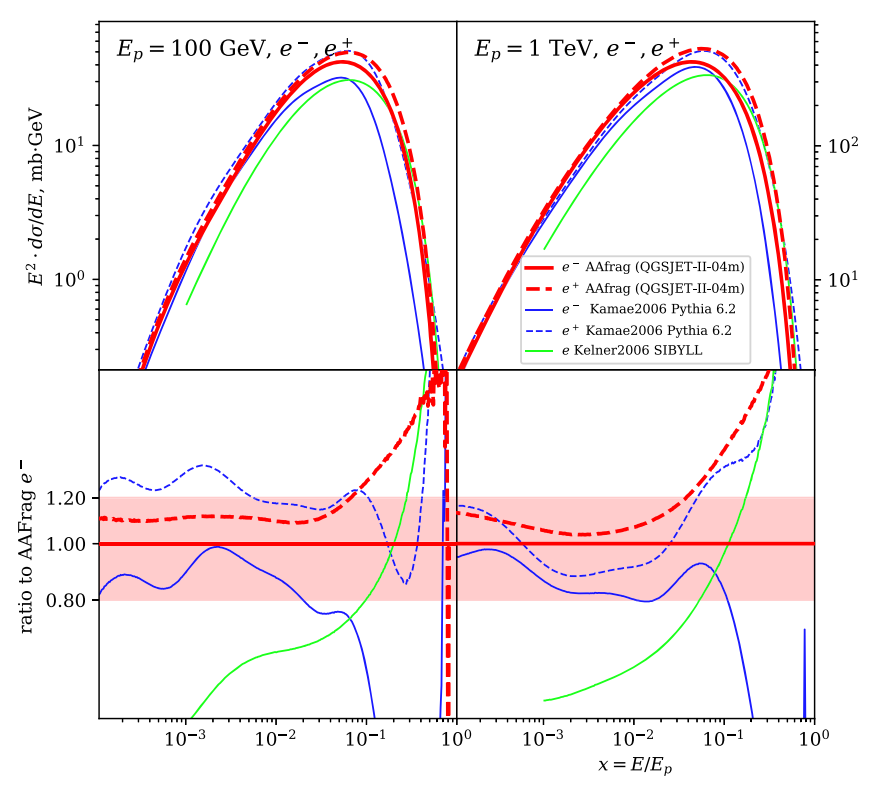

FIG. 6. Electron and positron spectra $E^{2} \mathrm{~d} \sigma / \mathrm{d} E$ as a function of the transferred energy fraction $x=E / E_{p}$, for $100 \mathrm{GeV}$ and $1 \mathrm{TeV}$ incident protons.

$$
I_{a}(E)=\int_{0}^{\infty} \mathrm{d} l n_{\mathrm{gas}} \int_{E}^{\infty} \mathrm{d} E^{\prime} \frac{\mathrm{d} \sigma_{a}}{\mathrm{~d} E}\left(E^{\prime}, E\right) I_{p}\left(E^{\prime}\right),
$$

where the $l$ integration is along a fixed line-of-sight and $n_{\text {gas }}$ denotes the number density of target protons. The cosmicray spectrum $I_{p}$ is expected to follow in most of sources roughly a power-law in momentum, modified by a highenergy cutoff which is often chosen as an exponential,

$$
I_{p}(p)=p^{-\gamma} \exp \left(-p / p_{0}\right)
$$

In the case of diffusive acceleration on nonrelativistic supersonic shocks, one expects $\gamma \simeq 2.0-2.2$ [24]. In the following examples, we will use $\gamma=2$ and use as energy of the cutoff $p_{0}=100 \mathrm{TeV}$. Additionally, we will impose a sharp low-energy cutoff at $E_{p}=4 \mathrm{GeV}$, since the AAfrag predictions are available only above this energy.

\section{A. Photon and neutrino spectra}

In view of the differences between the predictions of AAfrag and of the other parametrizations, for monoenergetic protons, we expect a corresponding spread both for the normalization and the shapes of the spectra of gamma-rays and neutrinos also for broad energy distributions of primary protons.

In Fig. 7, we compare the spectra of secondary gammarays, calculated using AAfrag, with the those obtained from the parametrizations. One can see that the overall normalization of the predicted gamma-ray fluxes varies within $\pm 25 \%$ in a wide energy range. In addition, for the parametrizations of Kelner et al. and of Kafexhiu et al. based on

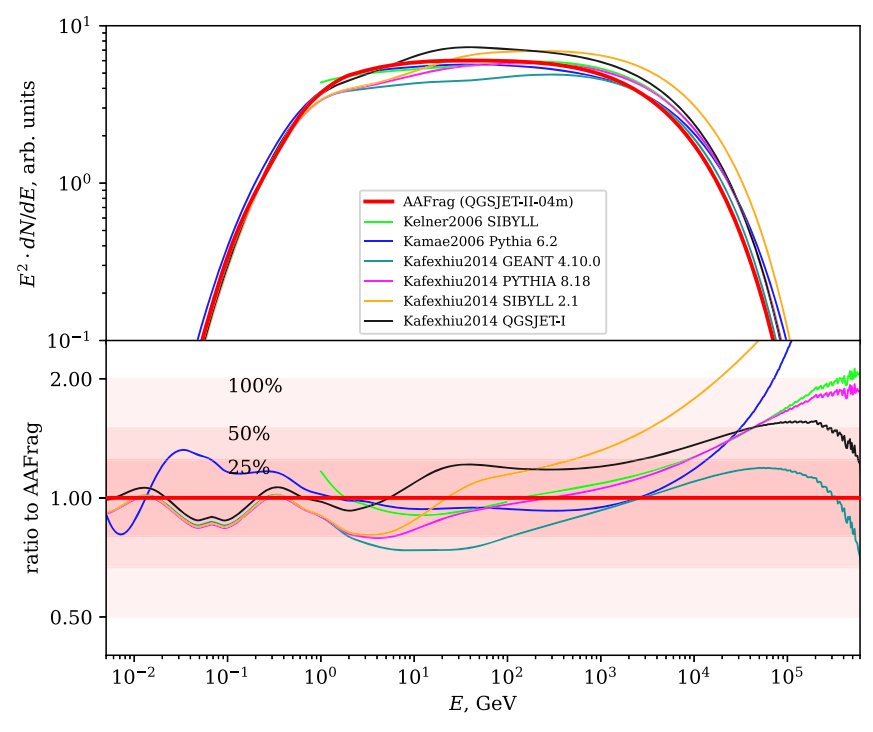

FIG. 7. Gamma-ray energy flux for a power law primary proton spectrum, with an exponential cutoff, $\propto 1 / p^{2} \exp \left(-p / p_{0}\right)$, $p_{0}=100 \mathrm{TeV}$. Upper panel compares the fluxes calculated with AAfrag using QGSJET-II-04m and the ones of [15] based on SIBYLL, [13] based on PYTHIA6.2, and [14] based on GEANT4.10.0, PYTHIA8.18, and QGSJET-I. Lower panel shows the ratio of the fluxes for the different parametrizations to the one of AAfrag.

GEANT4.10.0, we observe a slightly harder energy slope than for AAfrag. In the particular case of the parametrization of Kelner et al., this is explained by a too steep energy rise of the inelastic proton-proton cross section, which is at variance with the respective LHC data. On the other hand, the largest differences between the various predictions concern the spectral shape in the vicinity of the highenergy cutoff, where the spread reaches a factor of two. This is not surprising since the steep fall-down of the primary proton spectrum in the cutoff region greatly enhances the sensitivity of the results to very forward $\left(E_{\gamma} \rightarrow E_{p}\right)$ photon production in proton-proton interactions, for which substantial differences between the model predictions have been observed in the previous section (cf. Figs. 2 and 3). While the harder gamma-ray fluxes predicted by Kamae et al. is caused by deficiencies in the modeling of the diffractive scattering, the harder slope of the pion spectrum predicted by SIBYLL2.1 results in a corresponding rise of the photon spectrum in the parametrizations of Kelner et al. and Kafexhiu et al.. This may have important consequences for the modelling of the new population of PeV gamma-ray sources recently discovered by Tibet-AS $\gamma$ [25] and LHAASO [26].

In Fig. 8, we show the comparison of the all-flavor neutrino spectra obtained with AAfrag and the different parametrizations. Here, AAfrag differs from the other models even stronger. In particular, AAfrag predicts an up to $50 \%$ higher flux of neutrinos, over a broad energy range except the cutoff region, compared to the model of 


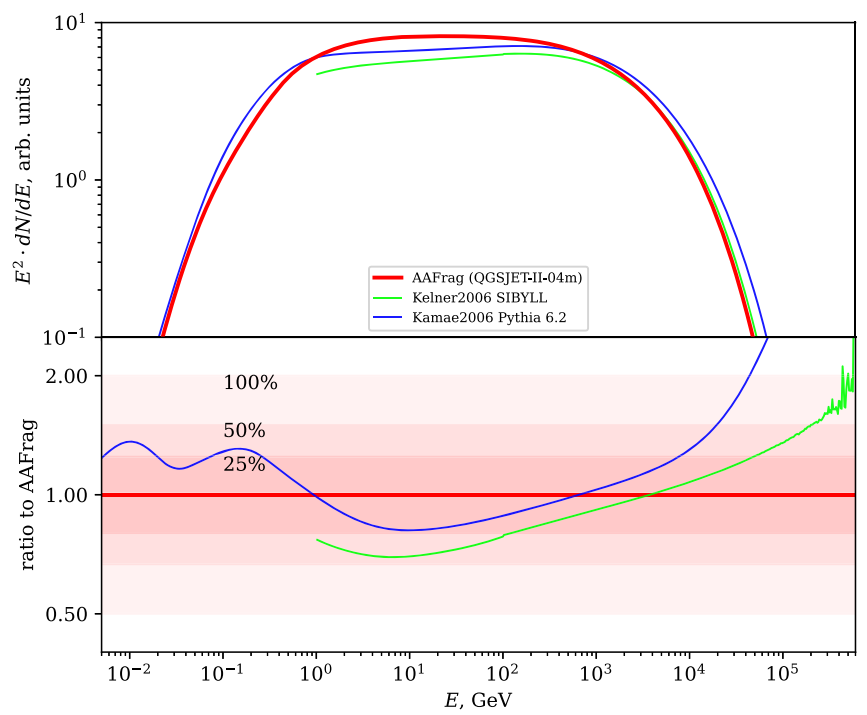

FIG. 8. All flavor neutrino flux for a power-law cosmic ray spectrum $\left(\propto 1 / p^{2} \exp \left(-p / p_{0}\right)\right)$.

Kelner et al. The agreement with Kamae et al. is better, with differences within 25\%. However, the high-energy cutoff in the neutrino spectra of Kamae et al. is much sharper. This implies that the model predictions for neutrino events might differ by up to a factor of two.

\section{B. Electron and positron spectra}

In the upper panel of Fig. 9, we show the electron and positron fluxes produced by the modelled power-law cosmic-ray spectrum and calculated with AAfrag and Kamae et al., together with the average electron-positron spectrum from Kelner et al. The lower panel shows the ratio of these spectra to the electron spectrum calculated with

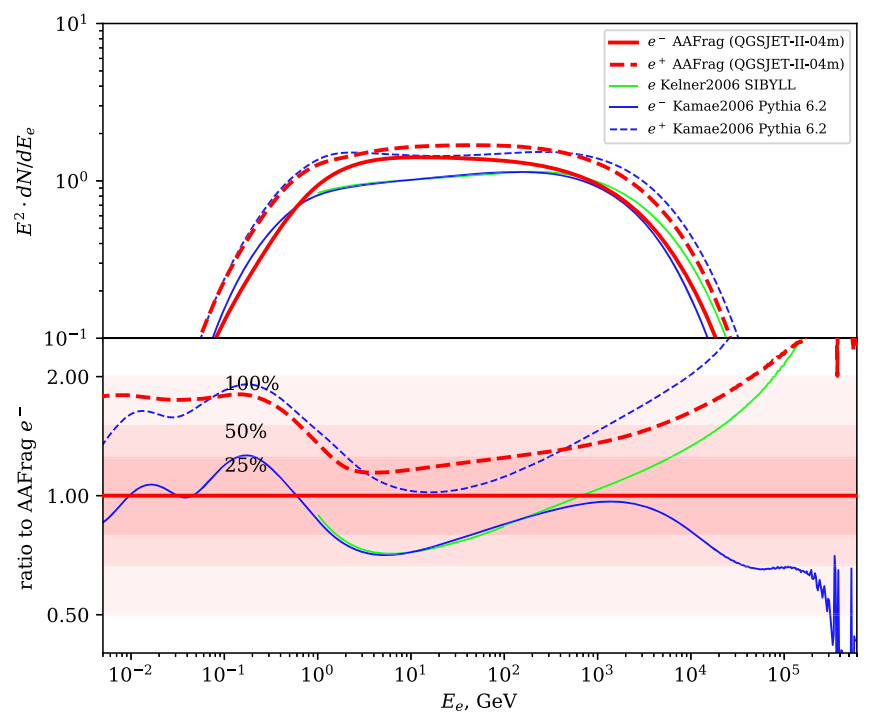

FIG. 9. Electron and positron fluxes for a power-law cosmic ray spectrum $\left(\propto 1 / p^{2} \exp \left(-p / p_{0}\right)\right)$.
AAfrag. We note first that both the normalization and the shape of the positron and electron spectra differ. While in AAfrag there are $\simeq 20 \%$ more positrons than electrons at few $\mathrm{GeV}$, this surplus exceeds a factor two close to the cutoff. As a result, the positron-electron ratio from hadronic interactions increases with energy toward the cutoff, an effect which is missed using the average electron-positron spectrum from Kelner et al.

\section{AAFRAGPY PYTHON PACKAGE}

So far we explored differences between the results of the various parametrizations, imposing a sharp low-energy cutoff at the primary particle energy $E_{p}=4 \mathrm{GeV}$, given that the energy range of AAfrag is constrained to $E_{p}>$ $4 \mathrm{GeV}$. Realistic astrophysical source spectra generally extend to lower energies and AAfrag cannot be directly used in such settings.

To overcome this limitation, we have developed a PYTHON package that implements a PYTHON interface to AAfrag and allows to complement AAfrag based calculations of particle production spectra with calculations based on parametrizations of low energy production cross sections. The package can be installed through the standard pip installer (pip install aafragpy). It provides several functions:

(i) The matrix of differential cross sections of secondary particle production in nuclear interaction for a given combination of primary and target nuclei for a given range of energies (function get cross section);

(ii) The differential spectrum of secondary particles produced in the interaction of a given primary spectrum with target nuclei (function get_spectrum).

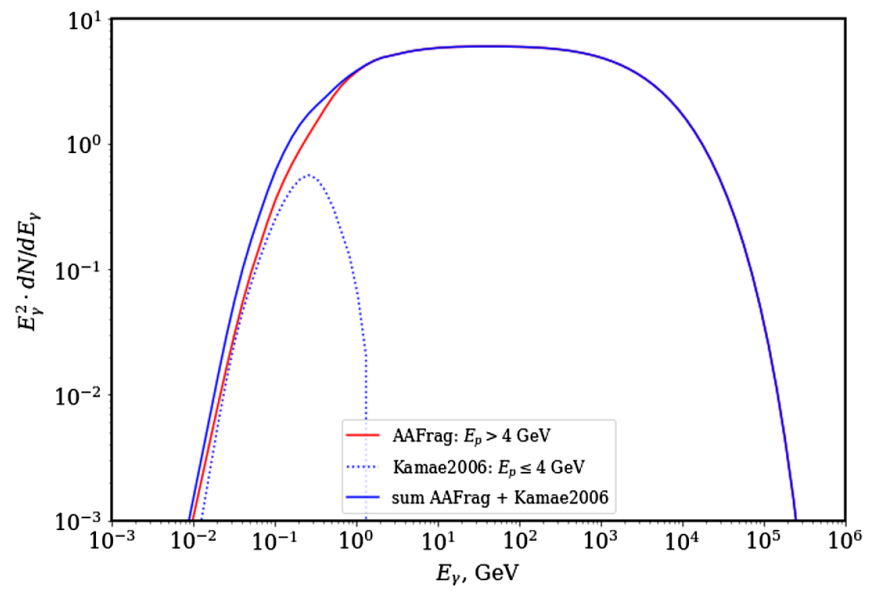

FIG. 10. Example of the spectrum of $\gamma$-ray emission from $p p$ interactions for an $d N / d p \propto p^{-2}$ powerlaw proton with cutoff on $p_{0}=100 \mathrm{TeV}$. The spectrum generated by AAfrag for proton energies $>4 \mathrm{GeV}$ is complemented by the spectrum calculated using the parametrizations of Kamae et al. [13] for $E_{p}<4 \mathrm{GeV}$. An url to interactive PYTHON notebook for the calculation of this spectrum can be found at GitHub page of the project: [27]. 
We have included two alternative parametrizations of production cross sections: those of Kamae et al. [13] and of Kafexhiu et al. [14]. The functions for the calculation of differential cross sections and spectra for these parametrizations are supplemented by_Kamae2006 and Kafexhiu2014 suffixes. Both parametrizations can $\bar{b}$ used only for the calculation of $p p$ cross sections.

Figure 10 shows an example of calculation of the spectrum of $\gamma$-rays from proton-proton interactions, for a power-law proton spectrum with exponential cutoff using such a combined approach. The AAfrag production spectrum calculated for protons with energies $E_{p}>4 \mathrm{GeV}$ is shown by the red solid line. The spectrum is complemented by the spectrum of $\gamma$-rays produced by protons with energies $E \leq 4 \mathrm{GeV}$, calculated using the Kamae et al. [13] parametrization, shown by the blue dotted line. The sum of the two spectra, shown by the blue solid line, corresponds to the total $\gamma$-ray spectrum from a proton distribution in a broad energy range.

\section{CONCLUSIONS}

We have compared the predictions of AAfrag for the spectra of secondaries produced in proton-proton collisions to those of three often used parametrizations. We have found considerable differences both in the normalization and the shape of the energy spectra, especially in the region of large energy transfer. A part of these variations is caused by the (unnecessary) procedure of fitting the results of QCD based event generators. More importantly, several parametrizations are based on outdated pre-LHC event generators. In the case of the energy spectra of photons, we have argued that LHCf results on the forward production of photons favor the use of the QGSJET-II-04m model on which AAfrag is based on. We have also stressed that the use of the average electronpositron spectrum misses the increase with energy of the positron-electron ratio in hadronic interactions.

The differences found in the normalization of the secondary spectra have important implications in the context of multi-messenger astronomy, in particular, for the prediction of neutrino fluxes based on gamma-ray flux measurements, or regarding the inference of the cosmic ray spectrum based on gamma-ray data.

We also present the easy-to-use PYTHON package aafragpy, which allows calculating the differential cross section and the spectrum of secondary particles produced in nucleus-nucleus interactions in the astrophysical sources. The package uses the calculations of the original AAfrag together with calculations performed by Kamae et al. [13] and Kafexhiu et al. [14] for low-energy secondary production.

\section{ACKNOWLEDGMENTS}

S. O. acknowledges support from the Deutsche Forschungsgemeinschaft (Project No. 465275045). S. K. acknowledges support from the Ministry of Science and Higher Education of the Russian Federation under project "Fundamental problems of cosmic rays and dark matter" No. 0723-2020-0040.
[1] M. Spurio, Probes of Multimessenger Astrophysics, Astronomy and Astrophysics Library (Springer, New York, 2018).

[2] M. Kachelrieß and D. V. Semikoz, Prog. Part. Nucl. Phys. 109, 103710 (2019).

[3] M. Aguilar et al. (AMS Collaboration), Phys. Rep. 894, 1 (2021).

[4] X. Bai et al., arXiv:1905.02773.

[5] B.S. Acharya et al. (CTA Consortium), Science with the Cherenkov Telescope Array (World Scientific Publishing, Singapore, 2018), ISBN: 978-981-327-008-4.

[6] M. G. Aartsen et al. (IceCube-Gen2 Collaboration), J. Phys. G 48, 060501 (2021).

[7] D. d'Enterria, R. Engel, T. Pierog, S. Ostapchenko, and K. Werner, Astropart. Phys. 35, 98 (2011).

[8] S. Ostapchenko, Phys. Rev. D 83, 014018 (2011).

[9] S. Ostapchenko, EPJ Web Conf. 52, 02001 (2013).

[10] M. Kachelrieß, I. V. Moskalenko, and S. S. Ostapchenko, Astrophys. J. 803, 54 (2015).

[11] M. Kachelrieß, I. V. Moskalenko, and S. Ostapchenko, Comput. Phys. Commun. 245, 106846 (2019).
[12] AAfrag: Interpolation routines for secondary production, available at https://sourceforge.net/projects/aafrag/.

[13] T. Kamae, N. Karlsson, T. Mizuno, T. Abe, and T. Koi, Astrophys. J. 647, 692 (2006); 662, 779(E) (2007).

[14] E. Kafexhiu, F. Aharonian, A. M. Taylor, and G. S. Vila, Phys. Rev. D 90, 123014 (2014).

[15] S. R. Kelner, F. A. Aharonian, and V. V. Bugayov, Phys. Rev. D 74, 034018 (2006); 79, 039901(E) (2009).

[16] M. Kachelrieß and S. Ostapchenko, Phys. Rev. D 86, 043004 (2012).

[17] N. N. Kalmykov and S. S. Ostapchenko, Phys. At. Nucl. 56, 346 (1993), https://ui.adsabs.harvard.edu/abs/1993PAN... $.56 . .346 \mathrm{~K} / \mathrm{abstract}$.

[18] M. Kachelrieß, I. V. Moskalenko, and S. S. Ostapchenko, Astrophys. J. 789, 136 (2014).

[19] O. Adriani et al. (LHCf Collaboration), Phys. Lett. B 703, 128 (2011).

[20] O. Adriani et al. (LHCf Collaboration), Phys. Lett. B 715, 298 (2012).

[21] O. Adriani et al. (LHCf Collaboration), Phys. Rev. D 94, 032007 (2016). 
[22] O. Adriani et al. (LHCf Collaboration), Phys. Lett. B 780, 233 (2018).

[23] F. Stecker, Cosmic Gamma Rays (Mono Book Corporation, Baltimore, 1971).

[24] A. R. Bell, Astropart. Phys. 43, 56 (2013).
[25] M. Amenomori et al. (Tibet ASgamma Collaboration), Phys. Rev. Lett. 126, 141101 (2021).

[26] Z. Cao, F. A. Aharonian, Q. An, Axikegu, L. X. Bai, Y. X. Bai, Y. W. Bao, D. Bastieri, X. J. Bi, Y. J. Bi et al., Nature (London) 594, 33 (2021).

[27] https://github.com/aafragpy/aafragpy. 